\title{
GPPS-CH-2020-0083
}

\section{Design Considerations and CFD for a Novel Rotor-Stator Cavity Test Rig}

\author{
Markus Wunderlich \\ RWTH Aachen University \\ wunderlich@ikdg.rwth-aachen.de \\ Aachen, NW, Germany
}

\author{
Prof. Manfred C. Wirsum \\ RWTH Aachen University \\ office@ikdg.rwth-aachen.de \\ Aachen, NW, Germany
}

\author{
Dr. Marco Konle \\ MTU Aero Engines \\ marco.konle@mtu.de \\ Munich, BY, Germany
}

\begin{abstract}
This paper presents the design and setup of a newly designed test rig for the investigation of flow and heat transfer phenomena in rotor-stator cavities. To focus purely on this complex interaction, the rotating cavity is setup without any channel mimicking a hot gas path. Rotating and stationary temperature measurements as well as PIV measurements will be conducted simultaneously to quantify the fluid-structure and heat transfer in a rotor-stator cavity, whose design is inspired by modern turbine designs of aircraft engines. The rotor consists of three parts, fitted together by a combination of interference fits and high-strength bolted connections. Results of FEM computations of the design of this rotor are presented. Regions of high stresses due to rotation and temperature loads are pointed out and operating limits are defined. Furthermore, the rolling bearing system is presented and the resulting rotordynamic behaviour is analysed. Radial and axial stiffness of the roller bearings themselves, as well as those of the housing and foundation are considered. Finally, results of preliminary fluid dynamic simulations are presented in order to underline the advantages of this test rig and the phenomena, which are expected in the experimental investigations.
\end{abstract}

\section{INTRODUCTION}

Flow phenomena and heat transfer mechanisms inside rotor-stator cavities and fully enclosed rotor cavities have been the main focus of many works in the past. Both fields of research and their applicability are vast and especially the latter can in general not be investigated, without considering at least general results of the first. Due to its nature, most components of turbomachines are exposed to multiple phenomena in rotor-stator and fully enclosed rotor cavity problems and specific research in this field is of special interest. Typical flow phenomena in such cavities have been known for a long time, since the well-known publications of (Batchelor, 1951) and (Stewartson, 1953), who both analytically investigated the flow over and inside rotating, infinitely large discs and presented analytical solutions to the reduced, axisymmetric Navier-Stokes-Equations. During the time of these publications, some controversies existed. On the one hand, Batchelor's solution describes a solid, nonviscous body of fluid, confined between the boundary layers at the enclosing rotating disks, which is rotating at some speed, slower than the disks themselves. On the other hand Stewartson found a solution, in which the tangential velocity of the fluid is zero everywhere, except in the boundary layer at the disks. Since then, many numerical and experimental investigation regarding flow phenomena inside such cavities have been published. (Itoh et al., 1992) performed early experiments of the flow inside an enclosed rotor-stator system. Velocity and Reynolds-Stress components where measured using traversable hot-wire probes. A large overview of early works on rotorstator systems is given in (Owen and Rogers, 1992). (Poncet et al., 2005) and (Da Soghe et al., 2012) both numerically and experimentally investigated the flow inside a rotor-stator cavity with different aspect ratios, rotational Reynolds numbers and with and without varying centripetal and centrifugal through-flow rates. The radial dimensions of their test rig was in the same order of magnitude as the newly designed rig. Axial dimensions in the new rig are three to six times larger. Extensive LDA measurements as well as static pressure measurements on the stationary wall have been performed, in order to characterize the flow inside the cavity. Their numerical simulations have been carried out on a sector model of the experimental domain with five elements in circumferential direction. The ratio of the radial cell height $\Delta r_{\mathrm{e}}$ to the height of 
the domain in radial direction $\Delta r_{\mathrm{D}}$ was $\Delta r_{\mathrm{e}} / \Delta r_{\mathrm{D}}=0.005$ to 0.007 . The cell length in axial direction $\Delta y_{\mathrm{e}}$, compared to the length of the domain $h$ was varied between $\Delta y_{\mathrm{e}} / h=0.0025$ to 0.04 . Turbulence modelling in both publications was done through a low Reynolds RSM model with the integration of specific effects of rotation on the Reynolds stress tensor, which was developed in (Elena and Schiestel, 1996). Both works present good agreement between the numerical approach and the conducted experiments, whereas the latter demonstrates the ability of the well-known low Reynolds $k$ - $\omega$-SST model to sufficiently capture flows over rotating disks. Their findings indicate the development of Batchelor- and Stewartsontypes of flow regions inside the cavity, depending on the amount and direction (centrifugal or centripetal) of the imposed through-flow. Based on these findings, (Da Soghe et al., 2012) use the $k-\omega$-SST model to extend and improve the capability of a 1D-In-House code for the estimation of core swirl rations in rotor-stator cavities, which was originally based on the experimental correlation from (Daily et al., 1964). (Debuchy et al., 2007) both numerically and experimentally investigated the influence of the geometric parameter $\lambda=\Delta r_{\mathrm{D}} / h$ on the flow characteristics inside a rotor-stator cavity. They used 3hole-probe pressure measurements and hot wire anemometry to compare experimental pressure and velocity data to the corresponding numerical computations, in which the RANS equations with the aforementioned $k$ - $\omega$-SST model where solved. Depending on $\lambda$, the preswirl of the flow, which enters the cavity on the stator side, is influenced strongly, leading even to the suppression of the Batchelor type flow inside the cavity at $\lambda=0$. (Hu et al., 2019) employed thermochromic liquid crystal (TLC) measurements and hot-wire anemometry (HWA) probes in order to quantify the heat transfer coefficient on the surface of a spinning disk in a rotor-stator cavity with centrifugal through flow. A positive correlation between the Nusselt number on the disk's surface and the through-flow coefficient $C_{w}=\dot{m} /(b \mu)$ is reported for values of the modified turbulent flow coefficient $\lambda_{\mathrm{t}, \mathrm{x}}=C_{w} / \mathrm{Re}_{\Theta}^{1.2}<0.0009$, whereas the correlation is negative when $0.0009<$ $\lambda_{\mathrm{t}, \mathrm{x}}<0.0028$. The fluid density $\rho$, rotational speed $\Omega$, maximum rotor radius $b$ and dynamic viscosity $\mu$ form the rotational Reynolds-number $\operatorname{Re}_{\Theta}=\rho \Omega b^{2} / \mu$. This work only employs velocity measurements at two radial positions, which may not be enough to fully quantify the resulting flow structures inside the cavity.

In the presented work, a novel test rig for the investigation of flow and heat transfer mechanisms to and from a rotor, whose geometry is inspired by modern turbine designs in aircraft engines, is presented. In this new rig, PIV and temperature measurements using thermocouples on the rotating disk and inside the cavity will be used to quantify the flow and temperature fields inside cavity and disk.

\section{METHODOLOGY}

The aim of this work is to present the design of a new test rig, which enables the investigation of flow and heat transfer phenomena inside a rotor-stator cavity, mimicking the internal turbine disk cooling geometry of a modern jet engine. Figure 1 depicts a cross section of the rig, including the rotor (purple), the bearing system (red) and the design of the inlet and outlet geometry, respectively.

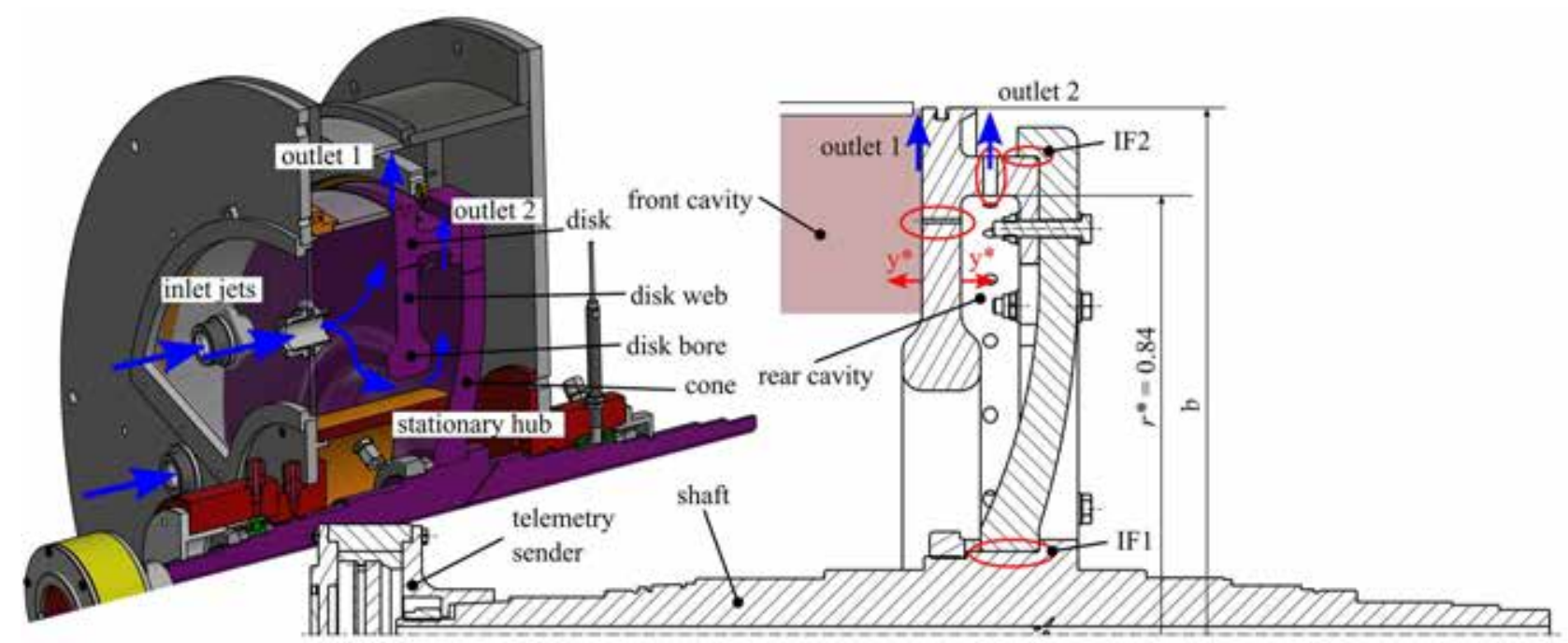

Figure 1: Left: 3D section of the test rig. Right: 2D section of the rotor (regions encircled in red show regions of high stress concentrations)

Air is blown into the rig via eight circumferential jets on a constant radius, impinging the rotating disk and influencing the flow field and heat transfer to and from the disk. Through-flow coefficients $C_{w}$ range between 15685 and 19321 , the rotational Reynolds-number $\operatorname{Re}_{\Theta}=\rho \Omega b^{2} / \mu$ falls in the range of $7.68 \cdot 10^{6}$ and $2.5 \cdot 10^{7}$. The geometric parameter $G=$ $h / b$ is 0.26 in the front and 0.12 to 0.15 in the rear cavity. A precondition of this rig is to use a rotor with similar heat capacity and conduction properties, as the actual engine. Therefore, instead of externally heating the rotor (which would 
take a long time and is a major challenge under high rotational speeds) and subsequently impinging it with coolant air, we reverse the thermal shock and impinge the rotor with hot air. The evolution of heat transfer coefficients on the rotor surface and the flow field in the front cavity will be experimentally determined by PIV and temperature measurements. Since the heat transfer here and in the actual engine is mainly convectively driven due to high velocities of the impinging jets and high relative velocities of the fluid and the rotor, the change of the direction of heat transfer and the adjustments of the inflow conditions is reasonable. Geometric dimensions are mostly adopted from an actual engine. The jet diameter $d_{\text {in }}$ of the injected coolant has been modified to keep Reynolds-number similarity and the injected coolant mass flow rate $\dot{m}_{\text {in }}$ constant, compared to that in the engine: $\dot{m}_{\mathrm{in}}=\dot{m}_{\mathrm{in}, \mathrm{E}}$. Air properties at inlet conditions, the bulk jet velocity $c$ and the inlet jet diameter $d$ define the jet Reynolds number at each inlet: $\operatorname{Re}_{\mathrm{in}}=\rho_{\mathrm{in}} c_{\mathrm{in}} d_{\mathrm{in}} / \mu_{\mathrm{in}}=4 \dot{m}_{\mathrm{in}} /\left(\pi d_{\mathrm{in}} \mu_{\mathrm{in}}\right)$. Consequently, the inflow jet diameter of the rig is: $d_{\mathrm{in}}=d_{\mathrm{in}, \mathrm{E}} \cdot \mu_{\mathrm{in}, \mathrm{E}} / \mu_{\mathrm{in}} \approx 1.76 d_{\mathrm{in}, \mathrm{E}}$. Inlet pressure and temperature of the coolant are kept well below engine conditions in order to simplify the design of the enclosure of the rotor and bearing system, since the mechanical demands here are high nevertheless. Pressure and temperature are below engine conditions. Consequently, the rotational speed is higher, in order to keep the rotational Reynolds-number $\operatorname{Re}_{\Theta}=\rho \Omega b^{2} / \mu$ constant: $\Omega=$ $\Omega_{\mathrm{E}} \mu_{\mathrm{in}} \rho_{\mathrm{in}, \mathrm{E}} /\left(\mu_{\mathrm{in}, \mathrm{E}} \rho_{\mathrm{in}}\right)$.

\section{FEM methodology}

The methodology of the finite element calculations, which were divided into calculations of the dynamic system and strength calculations of the rotor are described in the following paragraphs.

\section{Mechanical strength}

The assembly of the rotor consists of two interference fits, one tight fit, connecting shaft and cone, while the second, looser fit connects the cone and disk. In order to estimate the stress distribution inside the rotor, two cases, minimum and maximum interference at both respective surfaces are differentiated. Disk and cone are additionally connected via ten bolted connections with given bolt pretensions. The cone is axially secured via a shaft nut, which is tightly connected with the rotor. Static and dynamic strength verifications of the bolted connection were carried out using results of the strength calculations of the assembly and are not part of this paper. The material of the rotor is the commonly used, hardened and tempered steel 30CrNiMo8 with known yield strength $R_{\mathrm{p} 0,2}=1000 \mathrm{MPa}$. In order to get a conservative estimation of the actual stress distribution in the strength analysis, the material of the rotor is defined using bilinear isotropic hardening with a zero tangent modulus and the yield strength of the material. Consequently, no plastic hardening will occur. The loads are applied in different load steps, in order to ensure numerical stability and convergence. The load steps are: Interference fits, bolt pretension, temperature increase from ambient conditions to $40{ }^{\circ} \mathrm{C}, 0 \rightarrow 30 \%$ of the design rotational velocity $\Omega_{0}$, rotation $\left(0.3 \Omega_{0} \rightarrow \Omega_{0}\right)$, temperature increase to $140{ }^{\circ} \mathrm{C}$. The temperature distribution is assumed to be uniform at all times, since on the one hand, the temperature of the inlet fluid is not high even after the thermal shock $\left(T_{\mathrm{in}, 0}=140{ }^{\circ} \mathrm{C}\right)$ and on the other hand, the steady state temperature gradients, especially in regions of high mechanical stresses, do not yield excessive thermal stresses.

\section{Bearing system and the dynamic behaviour}

The rotor is supported by a fixed $\left(k_{\mathrm{B}, r, \text { fixed }}=2.25 \cdot 10^{8} \mathrm{~N} / \mathrm{m}, k_{\mathrm{B}, y}=36,500 \mathrm{~N} / \mathrm{m}\right)$ free $\left(k_{\mathrm{B}, r \text {,free }}=8.4\right.$. $10^{8} \mathrm{~N} / \mathrm{m}$ ) roller-bearing system. The listed radial and axial stiffness's $k_{\mathrm{B} . r}$ and $k_{\mathrm{B}, y}$ are directly taken from the specifications of the manufacturer and thus do not represent the actual stiffness of the structure, in which the rotor is supported. The supporting structures, as well as the housings of the bearings have been shown to potentially alter the total stiffness of the bearings significantly (Bogard et al., 2017; Gao et al., 2013). In order to get an estimation of the dynamic behaviour and to quantify the influence of the supporting structures of the bearings, several numerical simulations of the housings, as well as their connections to the supporting foundations have been carried out. A radial force $F_{r}$, with an angle of attack $\varphi$ from $-90^{\circ}$ to $+90^{\circ}$ in the radial-circumferential plane is applied to the shaft. The connection between the housings and their respective supports also plays a role, when considering radial displacements due to attacking forces at the bearings. Consequently, these connections are modelled frictional (friction coefficient $\mu_{\mathrm{F}}=0.15$ ), including four hexagon screws with a bolt pretension of $26,336 \mathrm{kN}$ each. Due to tightening of the bolts, a deformation $\Delta X_{\text {ref }}$ at the outer cylinder surface of the bearings occurs. This deformation is taken into account after applying $F_{r}$ and when evaluating the resulting deformation and thus, the radial stiffness $k_{r}$. We evaluate $k_{r}$ using two different approaches. A: Compute the radial deflection $\Delta X_{r, 1}$ of the centre (the arithmetic mean of every surface node) of the bearing surface before and after $F_{r}$ is applied (referred to as 'mean approach'). B: Compute the average radial deflection $\Delta X_{r, 2}$ of each individual surface node (referred to as 'edge approach'). 

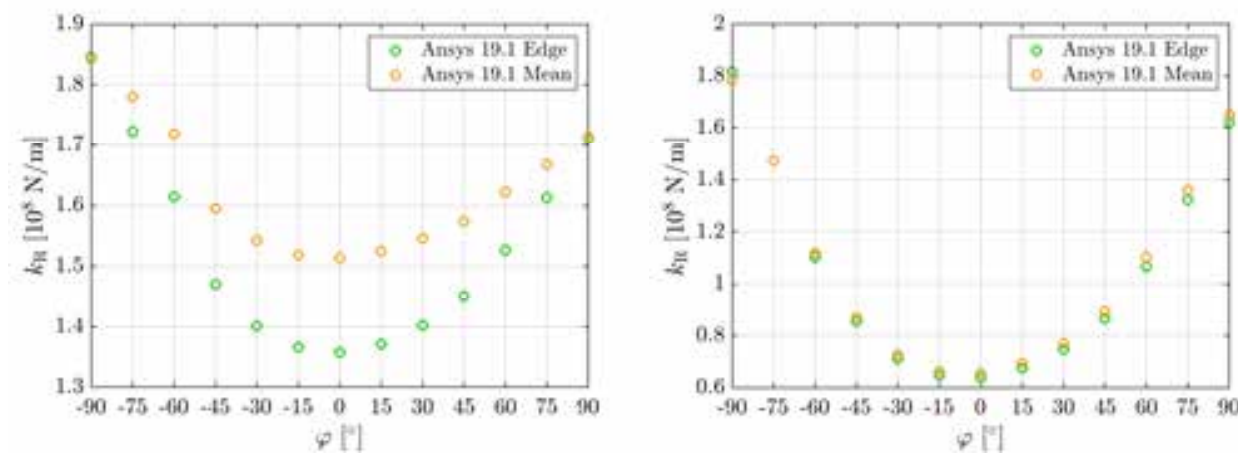

Figure 2: Total radial stiffness of the fixed (left) and free (right) bearing supports and housings

Subsequently the radial stiffness $c_{r}$ is the ratio of $F_{r}$ and the radial deflection $\Delta X_{r, i}$ in the direction of $F_{r}: k_{r}=$ $F_{r} /\left(\vec{e}_{r} \cdot \Delta X_{r, i}\right)$, where $\vec{e}_{r}$ is the unit vector in force direction. Figure 2 displays the radial stiffness of the fixed (left) and free bearing supports. The total radial stiffness is the serial superposition of the radial stiffness of the roller bearings and the radial stiffness of their respective housing: $k_{\mathrm{t}, r}=1 /\left(1 / k_{r}+1 / k_{\mathrm{B}, r}\right)$.

\section{STRENGTH ANALYSIS RESULTS}

Regions, where particularly high stresses are expected include wire holes through the disk, in order to direct the thermocouples to the telemetry system, ellipsoidal air outlet bores in the disk, the bore and the interference fits disk/cone and cone/shaft. These regions are highlighted in Figure 1. Figure 3 displays the distribution of negative normal stresses, in the region of the interference fit between cone and shaft (located at IF1 in Figure 1) in the case of maximum and minimum interference under all above describe loads.

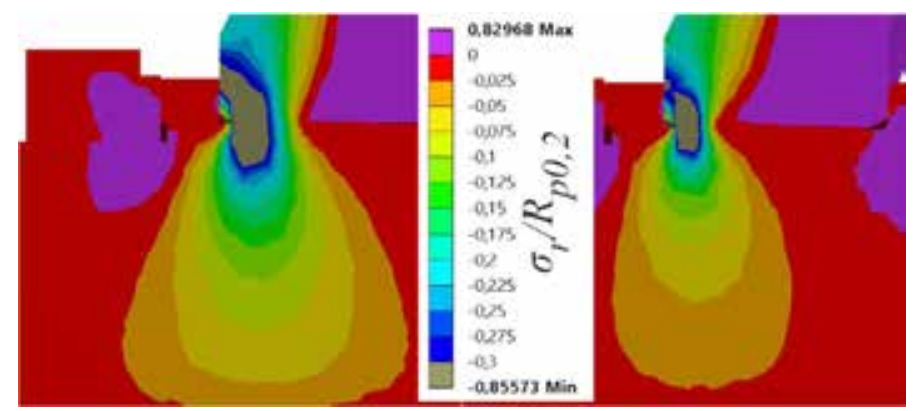

Figure 3: Normal stress distribution of the shaft/cone interference fit. Left: Maximum interference, Right: Minimum interference. Region in purple and red are positive normal stresses.

In both cases the cone lifts slightly at the shaft shoulder, but a negative radial stress and thus a save connection between shaft and cone remains in the region of the shaft nut. Radial pressure in the minimum interference case is $\mathbf{8 2 . 5 7} \%$, compared to the maximum case. The negative radial stress distribution inside the shaft expands further, if the fit is tight (max. interference), while the distribution inside the cone is identical. Figure 4 displays equivalent results for the interference fit between cone and disk (located at IF2 in Figure 1), in which case the main function, which is centering of the two parts, remains intact for minimum and maximum interference.

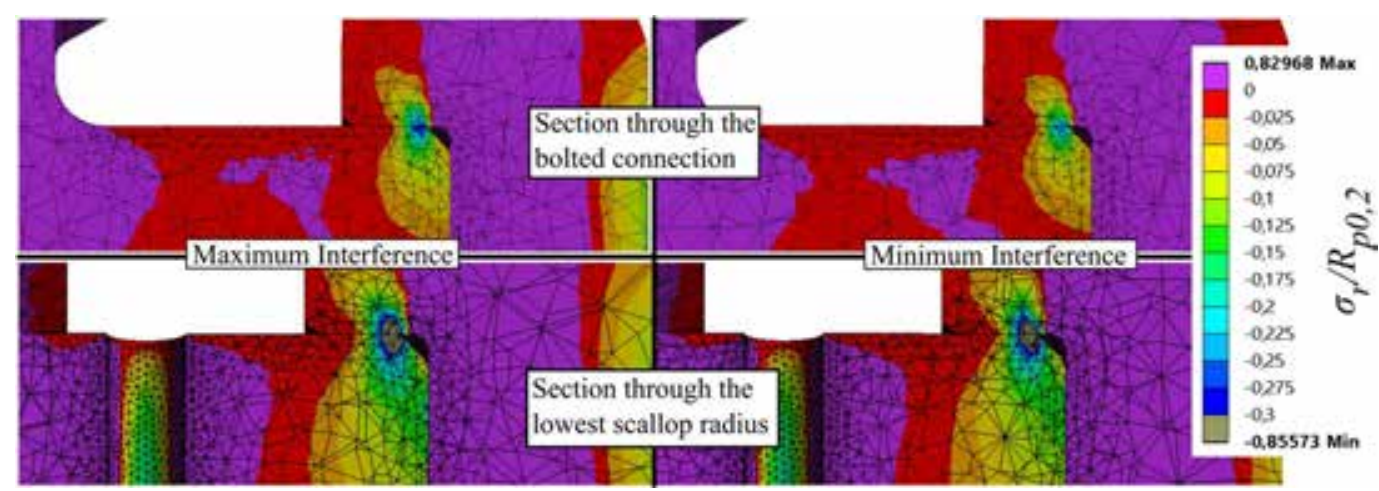

Figure 4: Normalized stress distribution in radial direction of the disk/cone interference fit. Left: Maximum interference, Right: Minimum interference. Purple regions are positive normal stresses. 
The top row displays a section view at the location of the bolted connection (section 2 in Figure 6), while a section through the lowest radius of a scallop (section 1 in Figure 6) is displayed in the bottom row. The pressure in the interference fit in the latter case is lower, compared to the first case. Here, less disk material is accelerated radially outward against the cone and thus reducing the pressure in the interference fit. Differences between maximum and minimum interference (left and right column in Figure 4) are limited to the vicinity of the fit-surface, the radial normal stress at minimum interference is about $91.7 \%$ compared to the maximum case. In all abovementioned cases, von-Mises stresses $\sigma_{\mathrm{v}}$, which are used to assess structural integrity, do not exceed the yield strength of the material. The selection of interference fit only slightly influences other regions outside, thus the case of minimum interference is chosen for all upcoming results.

In Figure 5 normalized von-Mises stresses in the elliptically shaped outlet holes at the rear cavity, inside the disk are displayed. The elliptical shape ensures lower stresses in the radial-circumferential plane (right), since the axial length of the holes is smaller, compared to round holes with equal areas. Consequently, more material in the axial direction is able to support the "overhang" of the disk and thus, hoop stresses are reduced. All von-Mises stresses stay well below the yield strength of the material. $\sigma_{\mathrm{v}}$ is generally highest, inside the wire holes through the web of the disk, but does not exceed $94 \%$ of the yield strength.

Finally, Figure 6 displays normal stress $\sigma_{n}$ distributions on the back side of the disk, which is bolted to the cone. The scalloped structure of this part of the disk was chosen, in order to eliminate hoop stresses in the bolt holes. The left side represents normal stresses after tightening of the bolts, while the right side is the distribution after the last simulation step, including rotation and thermal boundary conditions.

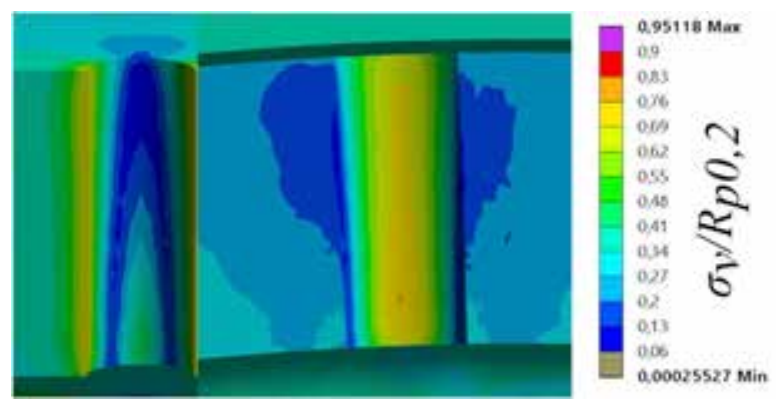

Figure 5: Normalized von-Mises stress distribution of the elliptical air outlets in the rear cavity. Left: Axial-radial section view. Right: Radial-circumferential section view.

In the first case, the normal stress on the disk is $31 \%$ higher on the edge of the bolt, which is radially inside, compared to the outer edge. This is a consequence of the axial stiffness of the flange, which increases with its radius. After applying rotation, an increase of the normal stress in the connection is evident, implying additional compression through deformation of disk and cone. The bolt force is consequently reduced by $12.3 \%$. All simulation results are reasonable and consistent with expectations and former experience.

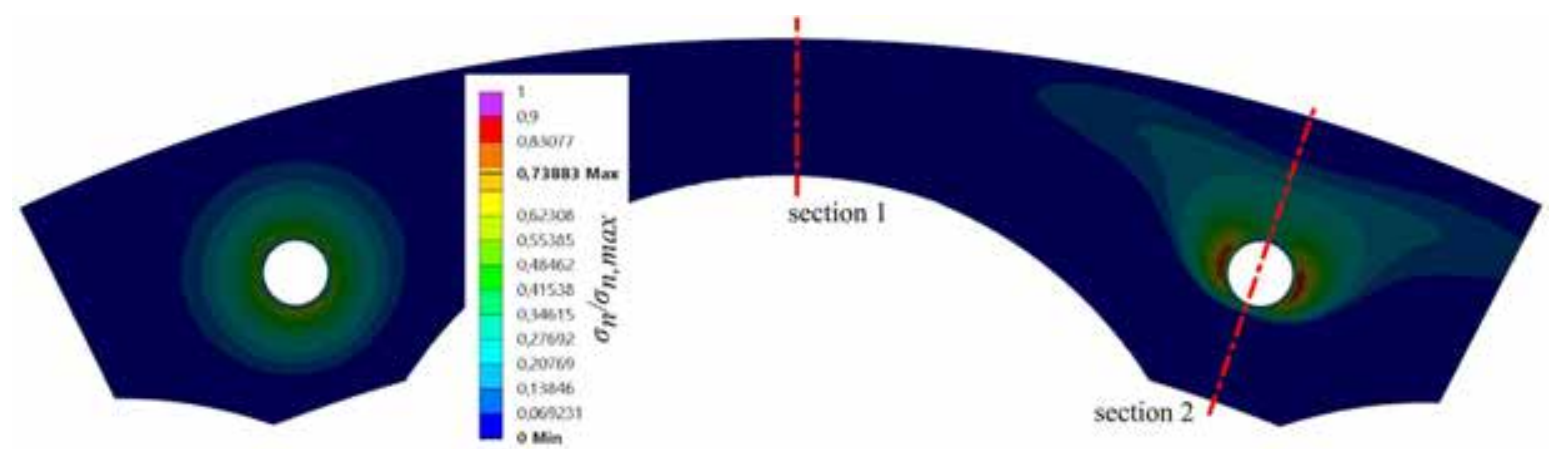

Figure 6: Normal stress distribution on the scalloped backside of the disk. Left: After tightening of the bolts. Right: After the last step, including rotation and thermal boundary conditions.

\section{DYNAMIC ANALYSIS RESULTS}

The results of four different modal analyses are described in the following paragraph. Here, we compare the resulting natural frequencies of the rotating system with and without thermally induced stress and temperature distributions in the rotor, using only the stiffness of each bearing as supplied by the manufacturer and - as a conservative approach - the 
minimum total stiffness $k_{\mathrm{t}, r}$ (Figure 2), respectively. At the shaft sections of the fixed and free bearings, bushing elements with velocity independent, constant radial stiffness were utilized to support the rotor. A thermal calculation was employed in order to calculate pre-stresses, which were applied as a boundary condition for the subsequent modal analysis. The temperature distribution in the rotor is the steady state temperature after the thermal shock. It was obtained by utilizing the so called TFEA-EXPO method (Diefenthal et al., 2017). The result of this method is the transient development of the rotor temperature and the respective heat transfer coefficients on its surface.

Figure 7 displays the resulting Campbell diagrams of all four modal analyses. M2 to M6 are the respective Eigenmodes, while excitation through unbalance is marked by the dashed line (EO1). The ratio $f / f_{0}$ represents Eigenfrequencies $f$, normalized by the design rotational speed (in Hz) $f_{0}$. When comparing both graphs $\left(k_{r}=k_{\mathrm{B}, r}\right.$ and $k_{r}=k_{\mathrm{t}, r}$, respectively), the significant influence of reducing the radial stiffness according to the aforementioned superposition of the housings and bearings becomes apparent: M2 is not affected, since the axial stiffness was not changed and this mode reflects the axial solid body movement of the whole rotor. M3 (backward whirling) and M4 (forward whirling) are tumbling modes of the disk and cone, with only slight participation of the shaft. In the left case in Figure 7, only M2 and M3 (regardless of thermal stresses) intersect with EO1 (unbalance) excitation around a normalized rotational speed of 0.7 ( 0.6 with thermal prestress). The rotational speed and vibration frequencies are normalized by the design rotational speed of the test rig $\Omega_{0}$. Due to the aforementioned reduction of $k_{r}$ (Figure 7, right side), modes M3 and M4 also intersect with EO1, since both, the frequencies, as well as the mode shapes change drastically, as displayed in Figure 8 and Figure 9. The color scale represents total deformation from zero (blue) to maximum (red). This dynamic behaviour would heavily impend the operation at nominal conditions, since the excitation lies very close to the nominal rotational speed.
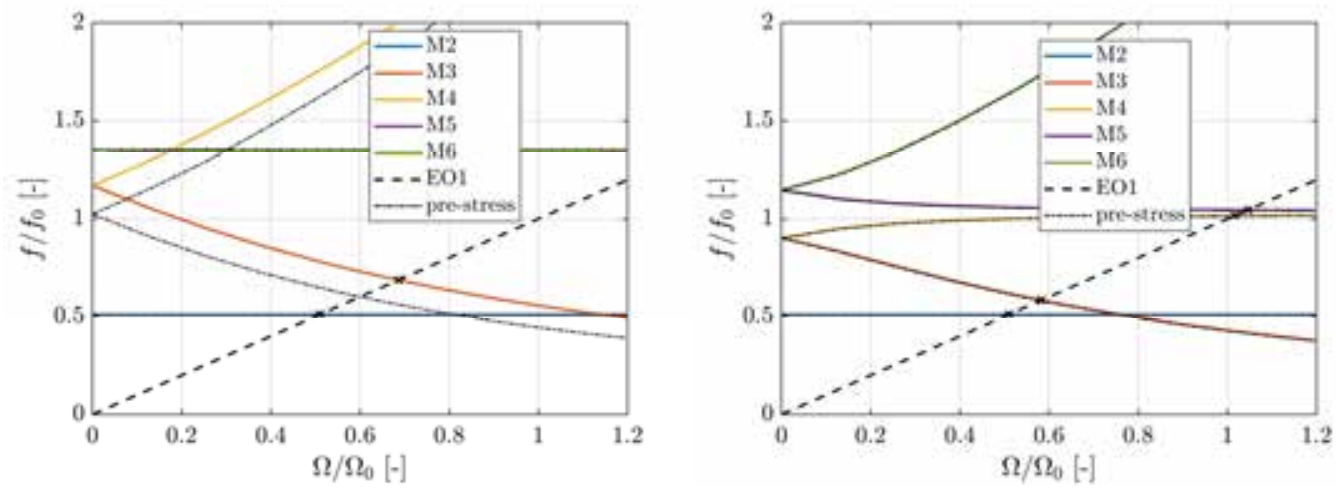

Figure 7: Campbell diagrams of the rotor. Left: $\boldsymbol{k}_{r}=\boldsymbol{k}_{\mathrm{B}, \boldsymbol{r}}$. Right: $\boldsymbol{k}_{\boldsymbol{r}}=\boldsymbol{k}_{\mathrm{t}, \boldsymbol{r}}$. Dash-dotted lines indicate
modal results when taking thermal pre-stresses into account.

As already mentioned above, Figure 8 and Figure 9 display the mode shapes of M3 through M6 at two different rotational speeds of the rotor $\left(0.128 \Omega_{0}\right.$ and $\left.1.197 \Omega_{0}\right)$. The top row in both figures depicts the case of $k_{r}=k_{\mathrm{B}, r}$, while the bottom row shows the shapes in case of $k_{r}=k_{\mathrm{t}, r}$. Contour colors represent total deflections of each mode shape. Since this is a modal analysis, the magnitude of the deflections are scaled arbitrarily, in order to showcase the shape of the modes. In the first case, independently of the rotational speed, M3 and M4 represent backward and forward whirling tumbling modes of the disk and cone, with little involvement of the shaft. As expected, due to the high radius and mass of the disk and thus, large influence of coriolis forces, the frequencies of those modes change drastically with the rotational speed of the rotor (Figure 7, left). M5 and M6 represent pure bending modes of the shaft, without any inclination of the disk or cone. Consequently, almost no influence of the rotational speed of the rotor, on the respective mode frequencies, is observed. The second case - with a significantly reduced radial stiffness of the bearing system - presents a different and very rotational speed dependent behaviour of all four mode frequencies and shapes, which is depicted in Figure 8 and Figure 9.

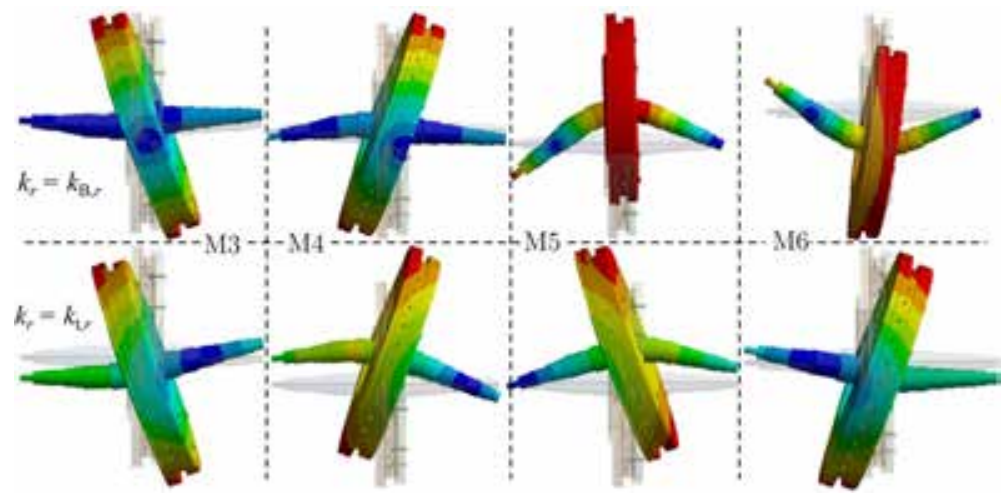


Figure 8: Mode shapes M3 through M6, $\Omega=0.128 \Omega_{0}$. Top row: $k_{r}=k_{\mathrm{B}, r}$, bottom row: $\boldsymbol{k}_{r}=k_{\mathrm{t}, r}$

At low rotational speeds (Figure 8, bottom row), M3 through M5 all incorporate some tumbling movement of the disk and cone. All respective frequencies are lower, when compared to the first case (Figure 7, right). When approaching higher rotational speeds (Figure 9, bottom rom), M6 mimics M5 of case one (forward whirling, tumbling mode), while M4 and M5 approach solid disk and cone shaft vibratory behaviour, as indicated by the color scale. Due to the reduction of radial bearing stiffness's, all mode frequencies are lower, hence promoting excitation of M4 and M5 at $\Omega=\Omega_{0}$ through unbalance. The above results lead to the closer examination of the radial stiffness of the bearing system, when accounting for the rotor housings, as well as the bearings themselves. Figure 10 (left) displays the meshed model of the complete rotating system, including the bearing housings, the support and the rotor itself. Since the above results lead to worrying possible excitations of eigenmodes, this system is subjected to a modal analysis as well. All solid body connections in this case are fixed, while for the connection between the shaft and both housings again bushings elements are utilized. In this case the actual radial (and axial) stiffness of the bearings are used directly and the radial stiffness of the housings and their respective supports is implicitly accounted for by the model itself.

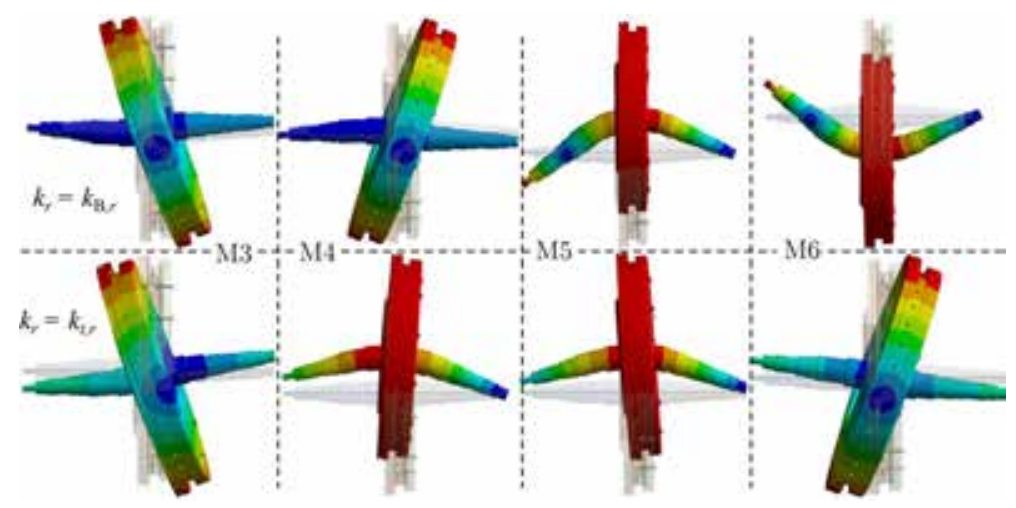

Figure 9: Mode shapes M3 through M6, $\Omega=1.197 \Omega_{0}$. Top row: $k_{r}=k_{\mathrm{B}, r}$, bottom row: $k_{r}=k_{\mathrm{t}, r}$

The respective Campbell diagram of this system is also shown in Figure 10. Eigenfrequencies from the above describe modal analysis of only the rotor and given bearing stiffnesses are represented by dash-dotted lines. As expected and in line with the results of a similar study (Gao et al., 2013), the bearing housings exert a softening influence on all eigenfrequencies, but not to the extent of the above described analyses. Especially at high rotational speeds, the influence of the supporting structures diminishes, compared to the stiffness of the bearings themselves. Also, mode shapes of all eigenmodes remain the same and do not change significantly with rotational speed. This analysis underlines the dynamic suitability of the bearing systems to support the rotor and ensure a safe operation at design rotational speed.
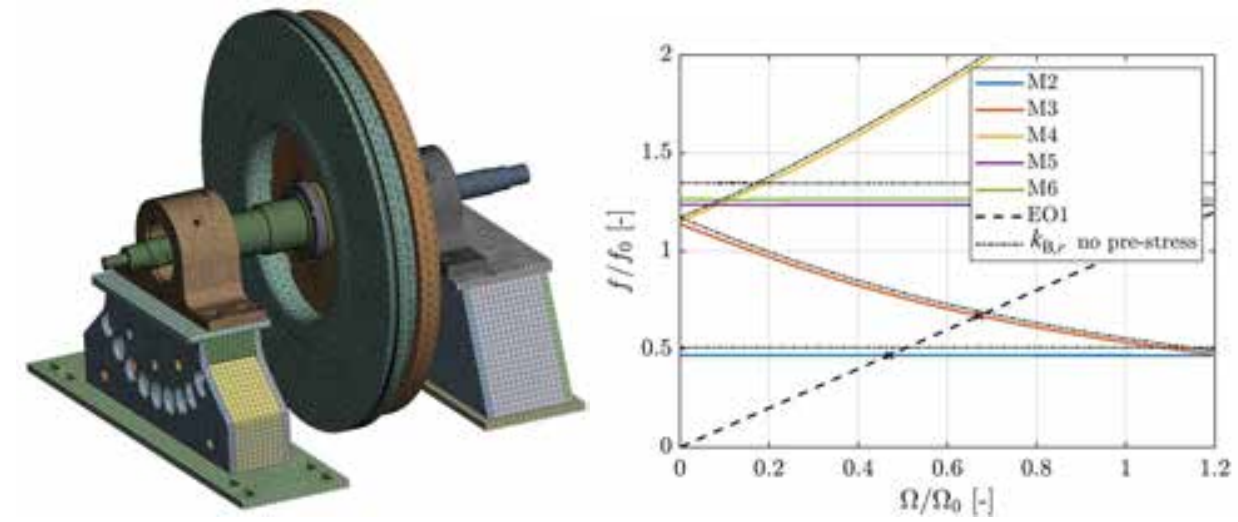

Figure 10: Left: FEM Mesh for the modal analysis of the rotor and bearing-housings. Right: Resulting Campbell diagram.

\section{CFD METHODOLOGY AND RESULTS}

In order to accurately capture all relevant flow phenomena inside the cavity, a detailed mesh analysis has been conducted. Table 1 summarizes relevant mesh parameters, of the investigated meshes. Additionally, two meshes with lower element counts have been considered, but were discarded later, since the 'coarse' mesh, which is shown here, already shows deficits.

Table 1: CFD mesh statistics 


\begin{tabular}{|c|c|c|c|}
\hline & Coarse & Medium & Fine \\
\hline Nodes & 4417213 & 8974073 & 15578487 \\
\hline Elements & 15059617 & 29928673 & 56013639 \\
\hline$\Delta y / h_{\text {Rear }}$ & 0.0667 & 0.0434 & 0.0334 \\
\hline$\Delta y / h_{\text {Front }}$ & 0.0267 & 0.0174 & 0.0134 \\
\hline
\end{tabular}

As a first step, we aimed to avoid rotor-stator interfaces and their common repercussions on the results. The least common multiple of 8 inlet jets (thus $45^{\circ}$ sections), 30 outlets $\left(12^{\circ}\right.$ sections) in the rear cavity and 10 scallops $\left(36^{\circ}\right.$ sections $)$ on the rotor is $180^{\circ}$, thus the computational domain represents exactly half of the physical domain of the test rig. Figure 1 visualizes the direction of the dimensionless axial coordinate $y^{*}=y / \mathrm{h}$ in the front and rear cavity, as well as the boundaries of the dimensionless radius $r^{*}=r / b . b$ is the maximum radius of the rotor and $h$ the axial gaps in the front and rear cavity, respectively. The coarse mesh is not able to capture the radial and circumferential velocity distributions in the front and rear cavity and at all radii in a physically reasonable manner. Figure 11 exemplarily shows distributions of the normalized radial velocity $c_{r}^{*}=c_{r} /(\Omega \mathrm{b})$ at several radii $r^{*}$ and circumferential angles $\Theta$, in both cavities and for all meshes. Black lines represent different time steps of the transient solution and the red line is the time averaged solution. Deficits of the coarse mesh are clearly visible in the boundary layer around $y^{*}=0.005$, since the unsteady change in velocity in this region is not physical.
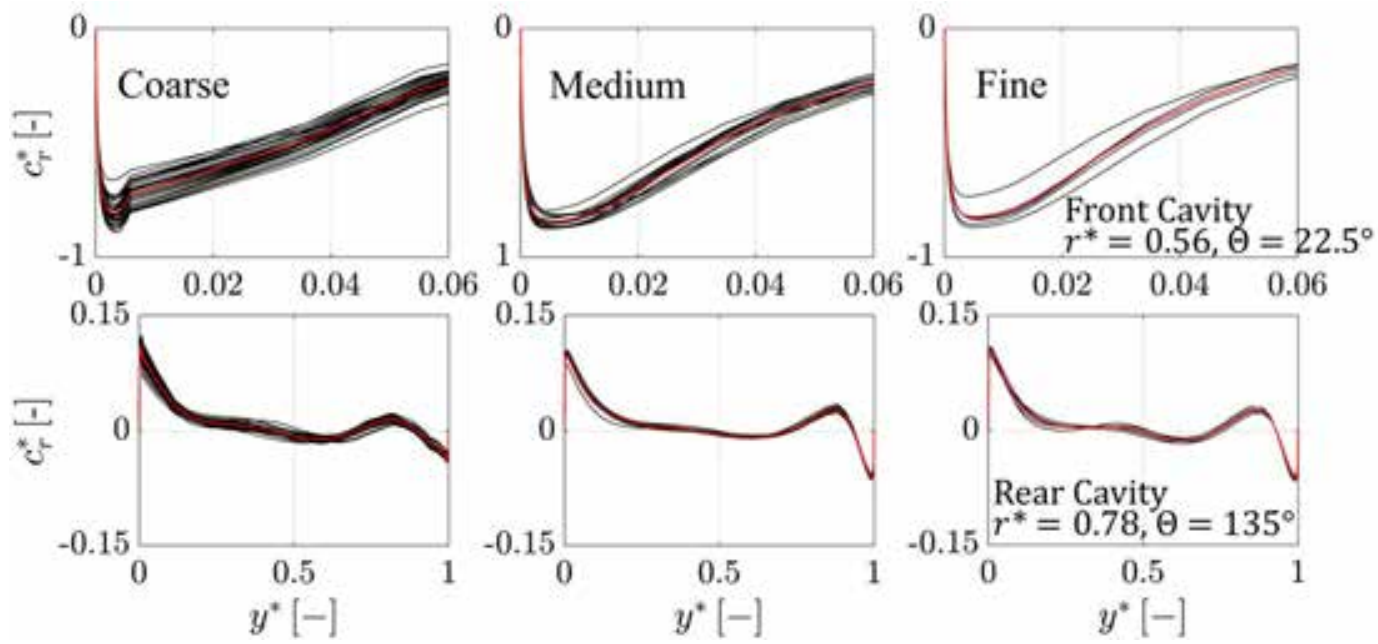

Figure 11: Normalized radial velocity in the front (top row) and rear cavity (bottom row). Left to right: coarse, medium and fine mesh.

Minor differences between medium and fine mesh do not justify the great additional computational costs of the latter. Consequently, the medium mesh is used for all future investigations of the flow field and comparisons with experimental data from the test rig. This mesh has also been used in the abovementioned coupled analysis in order to compute the transient temperature distribution of the rotor. The results reveal a strong transient behaviour of the flow field in the front cavity, at distances $y^{*}>0.5$, which is also not rotationally symmetric to a single jet (a $45^{\circ}$ section). In contrast to this, the flow field in the rear cavity is almost perfectly rotationally symmetric and does also not show much of a transient behaviour, which can for example be seen in the second row of Figure 11. Analysis of the transient behaviour at stationary points in both cavities with time steps of $1^{\circ}, 3^{\circ}$ and $5^{\circ}$ rotor rotation per step reveal significant frequency content in the flow field up to $10 \Omega_{0}$, thus a time step of $3^{\circ}$ is sufficient. In this region, Batchelor and Stewartson flow types are observed, depending on the radial and circumferential location, justifying further investigations of the flow field at different operating conditions, even though in this region no experimental measurements are planned. However, since the main scope of this work is the structural design and dynamic analysis of the rotor, an integral part of this test rig, detailed CFD results are not presented here. This part of the paper only highlights the importance of further CFD investigation, in order to develop a stable CFD model to which later experimental data can be compared to.

\section{CONCLUSIONS}

This paper demonstrates the suitability of the presented, newly designed test rig to conduct detailed experimental thermal and velocity measurements, in terms of structural integrity of the rotor, the dynamic behaviour of the bearing rotor- system and the developing fluid structures inside the cavity. The test rig can safely be operated at the design rotational speed and different flow structures, which are also commonly reported in the literature, are expected to form in the front cavity. Here, PIV measurements are going to be employed for the experimental investigations. The solid and fluid were 
successfully coupled, in order to get a first estimation of the temperature distribution (not shown in the paper) inside the rotor, which could then be used in order to access its influence on the dynamics of the test rig. Mechanical stresses inside the rotor during operation are sufficiently low, compared to the yield strength of the material and enable a safe operation of the rig. The dynamic analysis reveals a strong influence of the radial bearing stiffness on eigenmodes and eigenfrequencies, especially at very low radial stiffness's, which were deduced from a simple series connection of the bearing- and housing stiffness. A modal analysis of the rotor, including the bearing housings and supports reveals a slight reduction of eigenfrequencies, but not to the extent of the abovementioned ones. Unbalance excitation in this case should occur around $0.6 \Omega_{0}$ and above $1.2 \Omega_{0}$, also allowing a safe operation at design operating conditions of the rig. Further CFD simulations will employ a rotor stator interface, as mentioned above, similar to (Karnahl et al., 2012). This enables a $36^{\circ}$ sector in the rear cavity, while keeping a $180^{\circ}$ sector in the front and increasing the mesh density, while keeping the total cell count constant and computational costs reasonable.

\section{NOMENCLATURE}

\section{Latin Symbols}

$b \quad$ maximum rotor radius

c fluid velocity

$c^{*} \quad$ dimensionless fluid velocity

$C_{\mathrm{w}} \quad$ through-flow coefficient

$\vec{e}_{r} \quad$ unit vector in radial direction

$f \quad$ Eigenfrequency

$f_{0} \quad$ design rotational speed in $\mathrm{Hz}$

F $\quad$ Force

G geometric parameter

$h \quad$ axial domain width

$k_{y} \quad$ stiffness in axial direction

$k_{r} \quad$ stiffness in radial direction

$\dot{m} \quad$ imposed massflow

$m_{R} \quad$ rotor mass

$p \quad$ pressure $r \quad$ radial direction

$r^{*} \quad$ dimensionless radius

$\Delta r_{\mathrm{e}} \quad$ cell height in radial direction

$\Delta r_{\mathrm{D}} \quad$ radial domain height

$\Delta y_{\mathrm{e}} \quad$ cell height in axial direction

$T$ temperature

$t \quad$ time

$\Delta X_{\text {ref }} \quad$ initial deflection of the cylinder surface of the bearings inside the housings

$\begin{array}{ll}y & \text { axial direction } \\ y^{*} & \text { dimensionless axial coordinate }\end{array}$

\section{Greek Symbols}

$\begin{array}{ll}\beta & \text { core swirl ratio } \\ \beta^{*} & \text { core swirl ratio at zero through-flow } \\ \mu & \text { dynamic viscosity } \\ \mu_{\mathrm{F}} & \text { coefficient of friction } \\ \lambda_{\mathrm{t}} & \text { turbulent flow parameter } \\ \lambda_{\mathrm{t}, \mathrm{x}} & \text { modified turbulent flow parameter }\end{array}$

$\lambda$

$\rho$

$\sigma$

$\Theta$

$\Omega$

$\varphi$

$\begin{array}{ll}r & \text { radial direction } \\ \text { rear } & \text { rear cavity } \\ \mathrm{v} & \text { von-Mises stress } \\ \mathrm{HWA} & \text { Hot Wire Anemometry } \\ \mathrm{PIV} & \text { Particle Image Velocimetry } \\ \mathrm{Re} & \text { Reynolds number } \\ \mathrm{LDA} & \text { Laser Doppler Anemometry } \\ k-\omega-\mathrm{SST} & \text { turbulence model } \\ \mathrm{t} & \text { total } \\ \text { max } & \text { maximum value } \\ \text { M2-M6 } & \text { Eigenmodes } 2 \text { to } 6\end{array}$

\author{
geometric parameter \\ fluid density \\ mechanical stress \\ circumferential direction \\ rotational velocity \\ angle of attack with respect to the vertical \\ direction
}

M2-M6 Eigenmodes 2 to 6

\section{Indices and Abbreviations}

$\begin{array}{ll}\mathrm{B} & \text { bearing manufacturer quantity } \\ \mathrm{E} & \text { quantity in the engine } \\ \mathrm{EO} & \text { engine order } \\ \text { front } & \text { front cavity } \\ i & \text { node number } \\ \text { in } & \text { inlet quantity } \\ 0 & \text { design point } \\ \text { fixed } & \text { fixed bearing } \\ \text { free } & \text { free bearing } \\ \mathrm{n} & \text { normal direction }\end{array}$




\section{ACKNOWLEDGMENTS}

The authors would like to thank the Federal Ministry for Economic Affairs and Energy for the financial support of this research project. The authors would also like to gratefully acknowledge MTU Aero Engines, whose support was essential for the present investigations. Simulations were performed with computing resources granted by RWTH Aachen University under project rwth0403.

\section{REFERENCES}

Batchelor, G. K. (1951) 'Note on a Class of Solutions of the Navier-Stokes Equations Representing Steady

Rotationally-Symmetric Flow', The Quarterly Journal of Mechanics and Applied Mathematics, vol. 4, no. 1, pp. $29-41$.

Bogard, F., Murer, S., Rasolofondraibe, L. and Pottier, B. (2017) 'Numerical determination of the mechanical stiffness of a force measurement device based on capacitive probes: Application to roller bearings', Journal of Computational Design and Engineering, vol. 4, no. 1, pp. 29-36.

Da Soghe, R., Facchini, B., Innocenti, L. and Micio, M. (2012) 'Some Improvements in a Gas Turbine Stator-Rotor Systems CoreSwirl Ratio Correlation', International Journal of Rotating Machinery, vol. 2012, no. 2, pp. 1-9.

Daily, J. W., Asbedian, V. V., Ernst, W. D., Massachusetts Institute of Technology. Hydrodynamics Laboratory and Massachusetts Institute of Technology. Department of Civil Engineering (1964) Enclosed Rotating Disks with Superposed Throughflow: Mean Steady and Periodic Unsteady Characteristics of the Induced Flow [Online], Hydrodynamics Laboratory, Department of Civil Engineering, Massachusetts Institute of Technology. Available at https://books.google.de/books?id=EhYzMgAACAAJ.

Debuchy, R., Gatta, S. D., D'Haudt, E., Bois, G. and Martelli, F. (2007) 'Influence of external geometrical modifications on the flow behaviour of a rotor-stator system: Numerical and experimental investigation', Proceedings of the Institution of Mechanical Engineers, Part A: Journal of Power and Energy, vol. 221, no. 6, pp. 857-863.

Diefenthal, M., Łuczyński, P., Rakut, C., Wirsum, M. and Heuer, T. (2017) 'Thermomechanical Analysis of Transient Temperatures in a Radial Turbine Wheel', Journal of Turbomachinery, vol. 139, no. 9, p. 663.

Elena, L. and Schiestel, R. (1996) 'Turbulence modeling of rotating confined flows', International Journal of Heat and Fluid Flow, vol. 17, no. 3, pp. 283-289.

Gao, Y., Li, Z., Wang, J., Li, X. and An, Q. (2013) 'Influences of bearing housing deflection on vibration performance of cylinder roller bearing-rotor system', Proceedings of the Institution of Mechanical Engineers, Part K: Journal of Multi-body Dynamics, vol. 227, no. 2, pp. 106-114.

Hu, B., Li, X., Fu, Y., Zhang, F., Gu, C., Ren, X. and Wang, C. (2019) 'Experimental investigation on the flow and flow-rotor heat transfer in a rotor-stator spinning disk reactor', Applied Thermal Engineering, vol. 162, p. 114316.

Itoh, M., Yamada, Y., Imao, S. and Gonda, M. (1992) 'Experiments on turbulent flow due to an enclosed rotating disk', Experimental Thermal and Fluid Science, vol. 5, no. 3, pp. 359-368.

Karnahl, J., Wolfersdorf, J. von, Tham, K.-M., Wilson, M. and Lock, G. D. (2012) 'Computational Fluid Dynamics Simulations of Flow and Heat Transfer in a Preswirl System: Influence of Rotating-Stationary Domain Interface', Journal of Engineering for Gas Turbines and Power, vol. 134, no. 5, p. 52502.

Owen, J. M. and Rogers, R. H. (1992) 'Flow and Heat Transfer in Rotating-Disc Systems. Volume 1. Rotor-Stator Systems', Journal of Fluid Mechanics, vol. 241, pp. 724-725.

Poncet, S., Chauve, M.-P. and Schiestel, R. (2005) 'Batchelor versus Stewartson flow structures in a rotor-stator cavity with throughflow', Physics of Fluids, vol. 17, no. 7, p. 75110.

Stewartson, K. (1953) 'On the flow between two rotating coaxial disks', Mathematical Proceedings of the Cambridge Philosophical Society, vol. 49, no. 2, pp. 333-341. 